\title{
TRANSIÇÕES DA PRÁTICA PROFISSIONAL DA ENFERMAGEM NO ENFRENTAMENTO DO NOVO CORONAVÍRUS
}

Gerarlene Ponte Guimarães Santos, ${ }^{1,2}$ Rayla Maria Pontes Guimarães Costal ${ }^{1,2}$ Márcia Teles de Oliveira Gouveia ${ }^{2}$ Márcia Astrês Fernandes ${ }^{2}$ https://orcid.org/0000-0002-2531-5389 https://orcid.org/ 0000-0002-1794-119 https://orcid.org/0000-0002-2401-4947 https://orcid.org/0000-0001-9781-0752

Objetivo: Refletir sobre as transições da prática profissional da enfermagem no enfrentamento do novo coronavírus à luz da Teoria de transição de Afaf Meleis. Método: Trata-se de um estudo reflexivo fundamentado no referencial teórico da Teoria da Transição da enfermeira Afaf Ibrahim Meleis. Resultados: Observou-se que foram precipitadas as transições saúde-doença e organizacionais na prática da enfermagem para o enfrentamento do novo coronavírus, efetivamente em relação ao adoecimento e à reorganização dos serviços, adoção de políticas com implementação de novas práticas de enfermagem e tecnologias, desencadeando medo, insegurança e incertezas nos profissionais de enfermagem. Conclusão: A adoção de comportamentos e a inserção de novas habilidades para o enfrentamento da covid-19, permeado pelo contexto de mudanças, foram estratégias utilizadas na prática profissional de enfermagem, que favoreceu um processo de transição saudável.

Descritores: Prática Profissional; Riscos Ocupacionais; Cuidados de Enfermagem; Infecções por Coronavírus; Cuidado de transição.

\section{TRANSITIONS OF PROFESSIONAL NURSING PRACTICE IN COPING WITH THE NEW CORONAVIRUS}

Objective: To reflect on the transitions of professional nursing practice in coping with the new coronavirus in the light of Afaf Meleis's Transition Theory. Method: This is a reflective study based on the theoretical framework of the Transition Theory of the nurse Afaf Ibrahim Meleis. Results: It was observed that health-disease and organizational transitions were precipitated in nursing practice to face the new coronavirus, effectively in relation to illness and the reorganization of services, adoption of policies with the implementation of new nursing practices and technologies, triggering fear, insecurity and uncertainties in nursing professionals. Conclusion: The adoption of behaviors and the insertion of new skills for coping with covid-19, permeated by the context of changes, were strategies used in professional nursing practice, which favored a healthy transition process. Descriptors: Professional Practice; Occupational risks; Nursing care; Coronavirus infections; Transitional care.

\section{TRANSICIONES DE LA PRÁCTICA PROFESIONAL DE ENFERMERİA PARA HACER FRENTE AL NUEVO CORONAVIRUS}

Objetivo: reflexionar sobre las transiciones de la práctica profesional de enfermería para hacer frente al nuevo coronavirus a la luz de la teoría de transición de Afaf Meleis. Método: este es un estudio reflexivo basado en el marco teórico de la teoría de la transición de la enfermera Afaf Ibrahim Meleis. Resultados: Se observó que las transiciones organizacionales y de enfermedades de la salud se precipitaron en la práctica de enfermería para enfrentar el nuevo coronavirus, efectivamente en relación con la enfermedad y la reorganización de los servicios, la adopción de políticas con la implementación de nuevas prácticas y tecnologías de enfermería, lo que provocó miedo , inseguridad e incertidumbres en profesionales de enfermería.Conclusión: La adopción de comportamientos y la inserción de nuevas habilidades para hacer frente a covid-19, impregnada por el contexto de los cambios, fueron estrategias utilizadas en la práctica profesional de enfermería, que favorecieron un proceso de transición saludable.

Descriptores: Práctica professional; Riesgos laborales; Cuidado de enfermeira; Infecciones por coronavirus; Cuidado de Transición.

IUniversidade Estadual do Piaui. Parnaiba-PI, Parnaiba-PI, Brasil.

${ }^{2}$ Universidade Federal do Piauí. Teresina-PI, Brasil.

Autor Correspondente: Gerarlene Ponte Guimarães Santos Email:gerarleneg@gmail.com Recebido: 3/7/20 Aceito: 3/8/20 


\section{INTRODUÇÃO}

Em tempos de pandemia de SARS-CoV-2, vírus causador da doença COVID-19, os profissionais da saúde, em especial a equipe de enfermagem, buscam informações para se resguardarem de uma possivel infecção. A equipe de enfermagem está em constante exposição aos riscos ocupacionais por diversas doenças infecciosas, advindas da prática profissional, dentre as quais se destacam as infecções respiratórias, como a COVID-19. Uma grande proporção de casos confirmados está relacionada à exposição ocupacional ${ }^{1}$. No hospital de Wuhan, China, os profissionais de saúde foram reconhecidos como um grupo de alto risco para esta infecção, ocorrendo 40 (29\%) casos de contaminação entre profissionais da saúde, sendo que 31 (77,5\%) trabalhavam em enfermarias, sete $(17,5 \%)$ em pronto socorro e dois (5\%) na Unidade de Terapia Intensiva - UTI²

Com efeito, o surgimento do novo coronavírus propiciou mudanças na forma de pensar e agir da enfermagem, afetando, consequentemente, a prática do cuidado. Para a realização do cuidado em enfermagem, faz-se necessário repensar a prática profissional, e não apenas concebê-la como a prestação de um serviço a outrem, na qual o bem-estar do outro é visto como fundamental e primordial. Não obstante, inserir o autocuidado no mesmo patamar, visto que, em tempos atuais, ofertar o cuidado ao paciente exige destes cuidadores um olhar para si mesmos, tanto quanto o cuidado com o outro.

Assim sendo, a prática profissional da enfermagem consiste em um sistema composto por estrutura, processos e valores, que instrumentalizam e apoiam a equipe de enfermagem durante a prestação dos cuidados. O exercício da autonomia profissional, o controle sobre o ambiente de trabalho e o suporte organizacional podem apresentar diferenças entre as instituições de saúde ${ }^{3}$, entretanto, diante de uma pandemia, considerada um evento crítico, requer da equipe de enfermagem um envolvimento com a experiência de transição.

A transição ocorre quando há passagem de um estado estável a outro estável (lugar ou condição) e requer por parte da pessoa, a busca de novos conhecimentos, alteração do comportamento e mudança no seu self. Nessa perspectiva, com o fito de subsidiar o entendimento das transições ocorridas na prática profissional da enfermagem, buscou-se adotar como aporte a Teoria das Transições, que define a transição como um processo complexo que afeta várias dimensões, podendo acarretar mudanças na vida, saúde, relacionamentos e meio ambiente $^{4}$, podendo apresentar diferentes padrões, a saber: simples ou múltiplas, sequenciais ou simultâneas, relacionadas ou não ${ }^{5}$

Além disso, as propriedades das transições podem ser permeadas por consciencialização (referentes ao conheci- mento e à percepção sobre a experiência de transição), empenhamento (refere-se ao grau de envolvimento da pessoa no processo de transição), mudança (relacionadas aos eventos críticos ou desequilibrios que levam às alterações de ideias), diferença (consiste nas expectativas não atendidas ou divergentes), espaço temporal de transição (referentes ao fluxo do tempo), eventos e pontos críticos (associados ao aumento da conscientização para lidar com a experiência da transição). Quanto aos padrões de resposta, eles podem ser avaliados por indicadores de processo ou de resultados, sendo o primeiro referente à sentimentos de ligação, interação e estar situado, e o último, relacionado ao domínio de novas competências e à reformulação de nova identidade ${ }^{5}$.

Diante do exposto, percebeu-se uma lacuna de estudos relacionados as transições da prática profissional da enfermagem para o enfrentamento do novo coronavírus, na perspectiva de uma teoria de enfermagem. Para tanto, o presente estudo propõe refletir sobre as transições da prática profissional da enfermagem no enfrentamento do novo coronavírus, à luz da Teoria de transição de Afaf Meleis.

\section{MÉTODO}

Trata-se de um ensaio teórico-reflexivo construído por meio de leituras, discussões e reflexões realizadas no decorrer da pandemia pelo novo coronavírus, a partir de vivências da prática profissional de enfermagem. Adotou-se o referencial teórico da enfermeira egípcio-americana Afaf Ibrahim Meleis, denominado de Teoria das Transições, que a classifica de acordo com a natureza do tipo de transição que afetam as pessoas: desenvolvimental (referentes à mudanças no ciclo vital), situacional (relacionadas às situações que requerem redefinições de papéis), saúde-doença (ocorre quando há alteração no estado de saúde-doença, ou seja, adoecimento) e organizacional (representa mudanças em ambientes institucionais, alterando os aspectos políticos, sociais, econômicos e as dinâmicas organizacionais) ${ }^{4}$

A reflexão apresenta-se em dois eixos de análise: transições no processo saúde-doença e transições organizacionais da prática profissional da enfermagem para o enfrentamento do novo coronavírus.

Transições no processo saúde-doença da prática profissional da enfermagem para o enfrentamento do novo coronavirus

Essa transição é influenciada pela diversidade cultural e aparecimento de doenças ${ }^{4}$. Dessa forma, os profissionais da enfermagem, por atuar na linha de frente no combate à COVID-19, sofrem maior exposição, em virtude do manejo de pacientes com doenças respiratórias agudas. Em um estu- 
do prospectivo com 3.398 profissionais da saúde, a equipe de enfermagem foi a mais exposta ( $n=1.705,50,2 \%)$, a exposição ocupacional de alto risco a COVID-19, resultou em morbidade grave, hospitalização e absenteísmó. Em outro estudo realizado nos Estados Unidos com 9.282 profissionais da saúde com diagnóstico da COVID-19, houve relato de exposição no cuidado prestado ao paciente com COVID-19, a maioria dos profissionais da saúde não necessitou de internação, no entanto, alguns casos foram graves, resultando em óbitos em todas as faixas etárias ${ }^{7}$.

A exposição e contaminação da equipe de enfermagem podem ocorrer durante a realização dos cuidados prestados, seja na nebulização, indução de escarro, no auxilio a procedimentos que geram aerossóis e gotículas, como as intubações orotraqueais, traqueostomias e quaisquer outros procedimentos que abordem as vias aéreas.

O exercício profissional na enfermagem é exercido por três categorias: enfermeiro, técnico e o auxiliar de enfermagem. O conhecimento específico de cada profissional sobre sua função requer um atendimento efetivo e qualificado, pautado na valorização do cuidado a pessoa, a família e a comunidade, dentro dos preceitos legais, de forma a assegurar o bem-estar do outro, conforme a Lei no 7.498/86 e o Decreto no 94.406/87 ${ }^{8-9}$

Precipuamente, a equipe de enfermagem, em sua formação profissional, necessita de cinco anos para a formação do enfermeiro, dois anos para técnicos em enfermagem e um ano para auxiliares em enfermagem, para que estejam aptos a cuidar do outro e exercerem suas profissões nas mais diversas necessidades. Quiçá, situações incomuns podem ocorrer e requerem o gerenciamento deste cuidado, como mediante a disseminação de doenças infecciosas ainda não conhecidas ${ }^{10}$, mas que podem afetar o exercício da prática profissional da enfermagem com o surgimento de doença ocupacional.

Inclusive, a COVID-19 pode ser considerada a nova doença ocupacional que está se manifestando nesta década. Assim, todos os profissionais da área da saúde devem estar em alerta, tendo em vista o aumento explosivo de casos ocorridos na China e em outros países, em que o sistema de saúde foi sobrecarregado, medidas eficazes de saúde pública para contenção do SARS-CoV-2 estão sendo implementadas, como isolamento e distanciamento social, detecção precoce de casos e quarentena. Neste novo cenário, a equipe de enfermagem está no grupo de risco ocupacional e deve adotar boas práticas de controle de infecção․․

Não obstante, ao exercerem sua prática profissional, é necessário considerar a distância do ar expirado na realização de determinados procedimentos, como oxigenoterapia por cânula nasal ou por máscara de Venturi, Ventilação Não
Invasiva - VNI, nebulizações, aspiração orotraqueal, dentre outros $^{11}$

Cabe enfatizar as medidas de prevenção recomendadas pela Organização Mundial de Saúde - (OMS) a serem adotadas pela comunidade e pelos profissionais de saúde: higienizar as mãos com água e sabão ou álcool em gel; evitar o contato com olhos, nariz e boca; praticar a higiene respiratória ao tossir ou espirrar; usar máscaras; realizar higienização das mãos após descarte de máscaras; manter distanciamento social (mínimo de um metro) de indivíduos com sintomas respiratórios. Destaca-se também que são exigidas, para os profissionais de saúde, precauções adicionais, isto é, proteger-se e impedir a transmissão nos cuidados de saúde, uso de Equipamento de Proteção Individual (EPI) adequado, incluindo a seleção, o uso, a remoção e o descarte dos mesmos ${ }^{12}$

O uso de EPI passou a ser a maior segurança adquirida por todos os profissionais da saúde. No entanto, muitos não têm experiência com a paramentação e a desparamentação, proporcionando exposição e contaminação por SARS-CoV-2 e, por isso, um elevado número de profissionais com diagnóstico da Covid-19, alguns evoluindo com sindromes respiratórias grave, com necessidade de internação em Unidade de Terapia Intensiva (UTI) e por vezes, evoluindo para o óbito. Em um estudo realizado na Grécia envolvendo 66 profissionais da saúde com diagnóstico da Covid-19, 4,5\% apresentaram risco alto, 0,9\% risco moderado, e 0,8\% risco baixo ${ }^{6}$

Estes profissionais estavam acostumados com suas vestimentas hospitalares, sapatos confortáveis, máscaras cirúrgicas, lavagens das mãos, retirada de adornos e uso de luvas de procedimentos ou cirúrgicas em plantões de 06 horas, 12 horas, 24 horas ou mais.

Nesse sentido, questiona-se: o que mudou? A mudança está na forma de como cuidar de um caso suspeito ou confirmado do novo SARS-CoV-2. É necessário seguir as recomendações das autoridades de saúde para essa nova doença. O uso e a remoção adequada dos EPI's por todos os profissionais de saúde renovam a esperança de se cuidar com segurança. O uso de máscaras cirúrgicas, tão comuns nos ambientes hospitalares, foi abrindo espaço para outros equipamentos de proteção ao profissional, como as máscaras N95, a PFF2, macacão, aventais, óculos, protetor/visor facial, burca, touca, propé ou bota, luvas e respirador monitorizado (capuz, traqueia e motor com filtro). É necessário selecionar o EPI apropriado, bem como a maneira correta de usá-lo, removê-lo e descartá-lo ${ }^{10}$

Logo, em tempos de SARS-CoV-2, como estão as equipes de enfermagem, que transformam seus saberes em práticas para o bem estar físico, mental e social das pessoas sob seus cuidados? Estão vestidas de medos e receios em executar 
suas práticas. Continuam a dividir espaços em ambientes hospitalares, mas seguem imbuídos de inseguranças em cada novo turno de trabalho, bem como em seu retorno ao domicílio. Com efeito, não é o medo de não saber fazer, mas de positivar para COVID-19, de transmitir aos seus familiares, de não poder retornar para seus lares, de ficar longe dos que amam, de ficar isolado

Muitas informações sobre a transmissibilidade da COVID-19 chegam aos profissionais da saúde, por meio de artigos científicos, portal de notícias, sites oficiais de saúde, televisão, redes sociais, Whatsapp, e-mails, rádios, dentre outros. Acompanhar as notícias sobre a pandemia, através de informações do número de casos, óbitos, curas e quantidade de profissionais da saúde que adoeceram, foram curados ou morreram passou a ser frequente no meio dos mesmos. Contudo, vão mais além, querem saber sobre os estudos relacionados à COVID-19, sobre os tratamentos, vacinas e curas.

Pelos noticiários toma-se conhecimento do expressivo número de profissionais de enfermagem que adoecem e morrem por COVID-19 diariamente. Desse modo, aumenta-se o drama vivenciado pela categoria e cria um alerta sobre a prática profissional, levando à seguinte reflexão: como continuar a salvar vidas e estar bem consigo? Salvar vidas foi e é a grande responsabilidade frente ao ser humano que padece. Promover, prevenir, restaurar e reestabelecer a saúde do indivíduo, da família e da comunidade são ações exercidas por todos que trabalham na assistência ao paciente. Percebe-se, então, o que mudou: o autocuidado. Cuidar do outro sem estar paramentado adequadamente expõe os profissionais da enfermagem a um maior risco de infecção pelo novo coronavírus.

Transições organizacionais da prática profissional da enfermagem para o enfrentamento do novo coronavírus

Essa transição é precipitada por mudanças em ambientes institucional, social, político, econômico, alterações na estrutura ou nas dinâmicas organizacionais ${ }^{4}$. Antes da pandemia, para muitos profissionais, sair de casa para trabalhar, entrar no ambiente hospitalar, cuidar dos doentes e voltar para casa era uma rotina vivida com tranquilidade; realizavam de forma segura um cuidado voltado para recuperação e bem-estar do paciente mediante teoria e prática. Com a chegada do novo coronavírus, que tem infectado seres humanos em escala global, notou-se receio e medo ao invés da segurança do cuidado com o outro. Esta situação foi capaz de paralisar e/ou interferir na prática profissional da enfermagem, pois o zelo consigo mesmo entrou como protagonista do cuidado a ser prestado.

Assim, a vida segue com outro olhar: todos precisam im- plementar ações de isolamento/distanciamento social ou quarentena. O uso de máscaras estende-se a toda população. Ir ao supermercado, ao banco e à farmácia requer medidas de autoproteção. O uso de álcool gel, antes um cuidado assegurado aos profissionais de saúde, passou a ser de responsabilidade comunitária.

No diálogo com a teoria, entende-se que toda transição impõe mudanças e diferenças no antes e depois, bem como exige um evento com significados críticos em um espaço de tempo, sendo que essa passagem pode acontecer de forma salutar ou não, dependendo da resposta individual de cada pessoa, pois observa-se que compreende um período de vulnerabilidade ao ser experenciado no processo transicional. Para o entendimento das experiências vivenciadas pelas pessoas durante o processo de transição, faz-se necessário conhecer os condicionantes pessoais e da comunidade, os quais podem facilitar ou não o alcance de uma transição saudável, precisamente a reformulação de sua identidade, o domínio de novas habilidades e a mudança de comportamentos ${ }^{13}$

Dessa maneira, diante das mudanças impostas e dos sentimentos vivenciados, veio o apoio da sociedade aos profissionais de saúde, em especial, aos médicos e à equipe de enfermagem, que pela primeira vez, foram aclamados como heróis na luta contra uma doença, fazendo-os se sentirem estimados e respeitados ${ }^{1}$.

Nesse contexto, necessita-se repensar as políticas de saúde do trabalhador para o profissional de enfermagem para que elas diminuam a sobrecarga do profissional e garantam seu bem-estar ${ }^{14}$, desenvolvendo suas práticas profissionais com confiança, estabilidade e proatividade, percorrendo as transições de forma saudável.

De certo, o Conselho Federal de Enfermagem - COFEN com o objetivo de proteger a enfermagem buscou fornecer aparato científico aos profissionais para que possam continuar a exercer a profissão, de forma segura e proativa. Dentre as ações implementadas para o enfrentamento da COVID-19 está o apoio a toda a equipe de enfermagem que está na linha de frente do combate a esta pandemia, disponibilizando material educativo, a saber: cartilhas sobre as recomendações gerais para organização dos Serviços de Saúde e preparo das equipes de enfermagem, orientação sobre paramentação e desparamentação de EPI, uma série de vídeos educativos relacionados à COVID-1915.

\section{Contribuições para a Prática}

O estudo reflete a transição da prática profissional da equipe de enfermagem frente a pandemia por COVID-19, ampliando um olhar sobre o cuidado de si e do outro, por 
meio da Teoria de Transição de Afaf Meleis. As mudanças na prática profissional exigem do enfermeiro, técnico e auxiliar de enfermagem um significado crítico em um curto intervalo de tempo. Muitos não tiveram treinamentos/capacitações para o uso apropriado do EPI, ou seja, usá-lo, removê-lo e descartá-lo. Assim sendo, a teoria abordada permite uma articulação entre a teoria de enfermagem e a prática profissional, promovendo o alcance para a mudança de comportamentos e adaptação para novas habilidades em um período de incertezas, dúvidas e conflitos.

\section{CONCLUSÃO}

As transições no processo saúde-doença e as organizacionais foram precipitadas pelos profissionais de enfermagem no enfrentamento da covid-19. Utilizou-se de novas habilidades, modificações de comportamentos e reconstrução de uma identidade pessoal e profissional, resultando em significados especiais, atividades de autocuidado e criação de um contexto de cuidados com a saúde, construídos de forma criativa e dinâmica, com o intuito de impedir consequências negativas e favorecer resultados positivos na prática do cuidado de si e do outro.
Notadamente, observa-se adoção de comportamentos e inserção de habilidades, permeadas pelo contexto de mudanças, que favorecem um processo de transição saudável, e consequentemente, resultados efetivos da prática profissional de enfermagem.

Com efeito, os profissionais de enfermagem apresentam grande desafio em suas práticas profissionais e expectativas de exercerem suas atividades e não contraírem o coronavirus. De fato, o medo é real, devido aos casos assintomáticos. Estar junto do outro e manter o distanciamento são as novas ações implementadas pelos profissionais de enfermagem em seu ambiente de trabalho.

Contribuições dos Autores: Concepção e/ou desenho: Santos, Gerarlene Ponte Guimarães e Costa, Rayla Maria Pontes Guimarães. Redação do artigo: Santos, Gerarlene Ponte Guimarães e Costa, Rayla Maria Pontes Guimarães. Revisão crítica: Gouveia, Márcia Teles de Oliveira e Fernandes, Márcia Astrês. Redação final: Santos, Gerarlene Ponte Guimarães e Costa, Rayla Maria Pontes Guimarães. Aprovação da versão final: Gouveia, Márcia Teles de Oliveira e Fernandes, Márcia Astrês.

\section{REFERÊNCIAS}

1 Huh S. How to train health personnel to protect themselves from SARS-CoV-2 (novel coronavirus) infection when caring for a patient or suspected case, J Educ Eval Health Prof. 2020;17:1-6.

2 Wang D, Hu B, Hu C, Zhu F, Liu X, Zhang J, et al. Clinical characteristics of 138 hospitalized patients with 2019 novel coronavirus-infected pneumonia in Wuhan, China. JAMA. 2020;323(11):1061-9.

3 Maurício LFS, Okuno MFP, Campanharo CRV, Lopes MCBT, Belasco AGS, Batista REA. Professional nursing practice in critical units: assessment of work environment characteristics. Rev. Latino-Am. Enfermagem. 2017:25:e2854. 4 Meleis AI, Trangenstein PA. Facilitating transitions: redefinition of the nursing mission. In: Meleis AI, editor. Transitions theory: middle- range and situation-specific theories in nursing research and practice. New York: Springer Publishing Company; 2010. p. 65-71.

5 Meleis AI, Sawyer LM, Im EO, Hilfinger Messias DK, Schumacher K. Experiencing transitions: an emerging middlerange theory. Adv Nurs Sci. 2000;23(1):12-28.

6 Maltezou HC, Dedoukou X, Tseroni M, Tsonou E, Raftopoulos V. Papadima K, Mouratidou E, Poufta S, Panagiotakopoulos G, Hatzigeorgiou D, Sipsas N. SARS-CoV-2 infection in healthcare personnel with high-risk occupational exposure: evaluation of seven-day exclusion from work policy [published online ahead of print, 2020 Jun 29]. Clin Infect Dis. 2020; ciaa888.

7 CDC Covid-19 Response Team. Preliminary estimates of selected characteristics among health care personnel with COVID-19-United States, February 12-April 9, 2020. MMWR Morb Mortal Wkly Rep 2020;69: Epub July 22, 2020.
8 Stolarski CV, Teston V, Kolhs M. Conhecimento da equipe de enfermagem sobre suas atribuições legais. REME - Rev Min Enferm.200913(3): 327-336.

9 Silva RS, Santos DT, Carvalho SS, Lisboa ACFN. Código de Ética dos Profissionais de Enfermagem: uma pesquisa documental. Enfermagem em Foco 2012; 3(2):62-66.

10 Oh N, Hong N, Ryu DH, Bae SG, Kam S, Kim KY. Exploring nursing intention, stress, and professionalism in response to infectious disease emergencies: the experience of local public hospital nurses during the 2015 MERS outbreak in South Korea. Asian Nurs Res (Korean Soc Nurs Sci). 2017:11(3):230 6.

11 Ferioli M. Cisternino C, Leo V, Pisani L, Palange P. Nava S. Protecting healthcare workers from SARS-CoV-2 infection: practical indications. Eur Respir Rev. 2020;29(155):1-10.

12 World Health Organization (WHO). Rational use of personal protective equipment for coronavirus disease 2019 (COVID-19) [Internet]. Geneva: WHO; 2020 [cited 2020 Feb 27]. 7 p. Available from: https://apps.who.int/iris/bitstream/handle/10665/331215/ WHO-2019-nCov-IPCPPE_use-2020.1-eng.pdf

13 Meleis AI. Theoretical nursing: development and progress. 5th ed. Philadelphia: Wolters Kluwer/ Lippincont Williams \& Wilkins; 2012.

14 Oliveira DM, Alencar NMBM, Costa JP, Fernandes MA Gouveia MTO, Santos JDM. Afastamento do trabalho por transtornos mentais e comportamentais entre profissionais de enfermagem. Rev Cuid. 2019;10(2):1-10.

15 Santos V, Persegona M, de Souza E, Almeida W, Filete M, da Silva M. Comitê gestor de crise do coronavirus no âmbito do Cofen. Enfermagem em Foco, [S.L.], v. 1l, n. 2, jul. 2020. ISSN 2357-707X. 